\title{
Paruresis and Parcopresis in Social Phobia: a case report
}

\author{
Paruresis e parcopresis em fobia social: relato de caso
}

Paruresis and parcopresis are manifested by a fear and inability to either urinate or defecate in public restrooms when other persons are present or may enter the room. ${ }^{1}$ Only one third of the patients suffering from these disorders have had their symptoms recognized by a health care professional. Patients with these diagnoses avoid trips, sporting events, parties, invitations and others social situations. ${ }^{2}$ This attitude of avoidance associated with anticipatory anxiety of the behaviors limits the patient's social functionality. Therefore, both diseases should be categorized as forms of social phobia. ${ }^{2}$

\section{Case Report}

A 23-year-old male patient who began to experience difficulties in social situations when approximately 15 years old. Social exposure was avoided by the patient for fear of being criticized or ridiculed. Thus, the patient had a posture of avoidance of some situations such as public speaking, speaking up in a meeting, attending social gatherings and using private or public bathrooms. When it was not possible to avoid attending the events the patient showed anxiety symptoms (muscle tension, sweating, rapid breathing and heart pounding). Consequently, there was damage to the patient's social functioning. At the age of 20 , the patient showed a certain improvement in some social phobic symptoms except for paruresis and parcopresis which continued intense. These symptoms limited the patient's social life because of the difficulties in going places outside of his home. It was also difficult to receive visits because of the rituals associated with paruresis and parcopresis. The patient is only able to defecate and to urinate when no person is present at home. In extreme situations, the patient requests that the family leave home for a few minutes to accomplish these activities. The patient underwent a clinical evaluation to rule out gastrointestinal diseases. Laboratory, imaging and endoscopic tests were normal.
The patient was treated with paroxetine $40 \mathrm{mg}$ daily for 6 months. The pharmacological proposals led to a small improvement of the condition. Therefore, the patient was referred for cognitive behavior therapy (CBT) to enhance treatment. This approach was carried out for 6 months (one session per week) leading to a partial clinical improvement. The pharmacological treatment showed better response when associated with CBT.

\section{Discussion}

The purpose of this case report was to describe an unusual subtype of social phobia whose most important finding is the avoidance of using toilets. The case demonstrates a patient with social anxiety disorder in which the main manifestations are paruresis and parcopresis. In the past, there was doubt whether the toilet phobia was a form of disorder in the urinary or digestive tract or represented a feature of some psychiatric disorder. ${ }^{1}$ The central point of this differentiation is the presence of pronounced avoidance behavior and of negative evaluation (recognizing the people and ridiculing the act of using the toilet). Currently, these disorders fall under the category of social phobia. ${ }^{3}$ As paruresis and parcopresis have a low prevalence (approximately $3 \%$ ) in patients with anxiety disorders ${ }^{4}$ the diagnosis of these events is not easily achieved by health professionals. The main reasons for underdiagnosis are the false association with organic disease, the limited knowledge of the diseases on the part of health professionals and the difficulty for the patients to seek help. ${ }^{4}$ Treatment is hampered by the resistance of patients to recognize the problem and the limited therapeutic response in a proportion of cases. Pharmacological treatment recommends the use of selective serotonin reuptake inhibitors, $B$ - blockers and other agents. ${ }^{5}$ However, pharmacotherapy used in the treatment of social anxiety disorder has shown low efficacy 
for paruresis and parcopresis. ${ }^{5}$ Cognitive behavioral approaches are useful in treating these diseases. ${ }^{5}$ These social phobia presentations are sub-diagnosed therefore it is recommended to look for the toilet avoidance in all patients with this diagnosis.

Régis Eric Maia Barros

Faculdade de Medicina de Ribeirão Preto, Departamento de Neurosciences and Behavior Universidade de São Paulo (USP)

\section{Disclosures}

Régis Eric Maia Barros

Employment: Universidade de São Paulo (USP), Brazil.

* Modest

** Significant

*** Significant: Amounts given to the author's institution or to a colleague for research in which the author has participation, not directly to the author.

\section{References}

1. Malouff JM, Lanyon RI. Avoidant paruresis: An exploratory study. Behav Modif. 1985;9(2):225-34.

2. Vythilingum B, Stein DJ, Soifer S. Is "shy bladder syndrome" a subtype of social anxiety disorder? A survey of people with paruresis. Depress Anxiety. 2002;16(2):84-87.

3. Hammelstein P, Soifer S. Is "shy bladder syndrome" (paruresis) correctly classified as social phobia? J Anxiety Disord. 2006;20(3):296-311.

4. Hammelstein P, Pietrowsky R, Merbach M, Brähler E. Psychogenic urinary retention ("paruresis"): diagnosis and epidemiology in a representative male sample. Psychother Psychosom. 2005;74(5):308-14.

5. Kaufman KR. Monotherapy treatment of paruresis with gabapentin. Int Clin Psychopharmacol. 2005;20(1):53-5. 